\title{
A rare case of CNS tuberculosis with pregnancy
}

\author{
Sanjay Singh $^{1 *}$, Tony Jose ${ }^{1}$, Puneet Saxena ${ }^{2}$
}

\author{
${ }^{1}$ Department of Obstetrics \& Gynaecology, Armed Forces Medical College, Pune-411040, Maharashtra, India \\ ${ }^{2}$ Department of Respiratory Medicine, Armed Forces Medical College, Pune-411040, Maharashtra, India
}

Received: 31 March 2015

Accepted: 09 May 2015

\author{
*Correspondence: \\ Dr. Sanjay Singh, \\ E-mail: drsanjaysingh@gmail.com
}

Copyright: ( $\odot$ the author(s), publisher and licensee Medip Academy. This is an open-access article distributed under the terms of the Creative Commons Attribution Non-Commercial License, which permits unrestricted non-commercial use, distribution, and reproduction in any medium, provided the original work is properly cited.

\begin{abstract}
Whereas pelvic tuberculosis leading to infertility is quite common in female population of developing countries, Central Nervous System (CNS) tuberculosis (TB) with pregnancy is a rare entity. Most of the information on this subject is based on sporadic case reports only. Most of the earlier reports suggest very high adverse outcome of CNS Tuberculosis in pregnancy. We are presenting a case of CNS Tuberculosis which was diagnosed timely and managed appropriately in our institute with a favourable outcome, thus highlighting the importance of early diagnosis and treatment.
\end{abstract}

Keywords: Tuberculosis, Central nervous system, Meningitis, Pregnancy, ATT

\section{INTRODUCTION}

Tuberculosis is a common infection in developing countries. Only $1 \%$ of these cases develop CNS tuberculosis. TB meningitis is the most devastating form of extra-pulmonary tuberculosis with very high mortality and morbidity. The initial clinical manifestation of TB meningitis may mimic normal pregnancy symptoms leading to delay in diagnosis resulting in complications to the mother as well as to the fetus. ${ }^{1-5}$ High index of suspicion is thus a key factor in its timely diagnosis. Timely diagnosis and appropriate institution of treatment resulted in favorable pregnancy outcome.

\section{CASE REPORT}

A 23 years old G2P1L1 lady, educated up to fifth standard, a home maker of low socioeconomic status, was admitted at 33 weeks Period Of Gestation (POG), with complaints of 5-6 episodes of vomiting per day and headache of fifteen days duration. She had been having fever with chills and rigor for three days and photophobia for one day. She had been feeling lethargic and her appetite had gone down for the last one month. There was no history of cough, chest pain, increased frequency of micturition, dysuria, head injury or convulsion. She didn't offer any complaints of pain abdomen or foul smelling vaginal discharge. She had perceived adequate fetal movements. Her last menstrual period was on 10 June 2014 making her expected date of delivery 17 March 2015. Her earlier cycles were regular. She had a full term normal vaginal delivery 3 years ago. She was a booked case and the index pregnancy was a spontaneous conception, confirmed by first trimester sonography. Her blood group was A positive. Sugar profile was normal. Screening test for hepatitis B, syphilis and HIV were negative. Second trimester sonography confirmed the dating and ruled out any obvious congenital anomalies. She was diagnosed to have Tuberculous Meningitis (TBM) one year back for which she was put on Anti Tubercular Treatment (ATT). However she defaulted and stopped ATT after two months against medical advice. 
On general examination her BMI was $21.4 \mathrm{Kg} / \mathrm{m}^{2}$. She was found drowsy and febrile $\left(100.8^{\circ} \mathrm{F}\right)$. There was no obvious pallor and lymphadenopathy. The cardiovascular and respiratory system was essentially normal. On CNS examination - Glasgow Coma Scale (GCS) was E4V4M6 with positive neck rigidity and Kernig's sign. There was no sensory motor deficit and plantar reflex was bilateral flexor. All the peripheral jerks were normal. Per abdominally fundal height corresponded to 32-34 weeks of gestation with normal fetal heart rate. Fundoscopy suggested bilateral papilloedema.

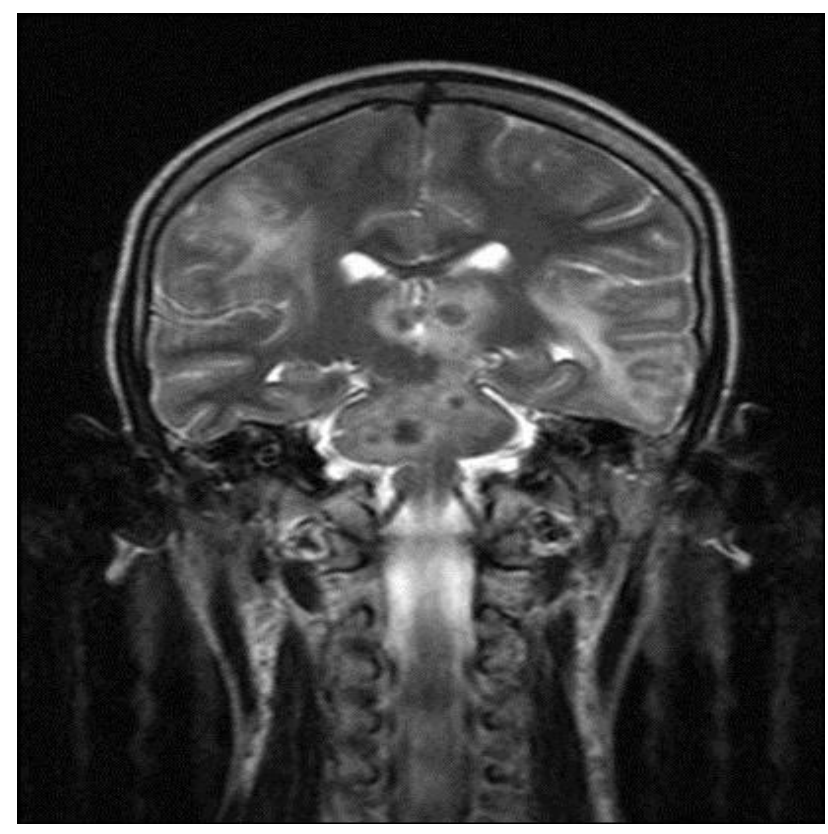

Figure 1: MRI brain showing multiple inflammatory granulomas (most probably Tuberculomas) in bilateral cerebral hemispheres, thalami, brain stem and cerebellar hemispheres.

Keeping cerebral malaria, meningitis and dengue in mind relevant investigations were done including lumbar puncture. On hematological evaluation hemoglobin was $11 \mathrm{gm} / \mathrm{dl}$ with TLC $-8400 / \mathrm{mm}^{3}$, P50 L40 M9 E1 B0 and raised ESR (70 mm/hour). No malaria parasite or toxic granules were seen on peripheral smear. Paracheck for malaria and dengue serology was negative. Liver function test, urea, creatinine and serum electrolytes were normal. Urine analysis was normal. CSF obtained on lumbar puncture had following characteristics-normal pressure, clear in appearance, TLC - 25 cells $/ \mathrm{mm}^{3}$, DLCpolymorphs - $40 \%$ lymphocytes $-60 \%$, red blood cells nil, sugar - $41.6 \mathrm{mg} / \mathrm{dl}$, protein - $87.4 \mathrm{mg} / \mathrm{dl}$, chloride $721.0 \mathrm{mg} / \mathrm{dl}$, adenosine deaminase (ADA) levels high (28.34IU/L). CSF culture was negative for acid fast bacilli. X-ray chest (done with abdominal shield) was normal. MRI brain (Figure 1) suggested multiple inflammatory granulomas (most probably tuberculomas) in bilateral cerebral hemispheres, thalami, brain stem and cerebellar hemispheres. She was diagnosed as a case of relapse of TBM and was put on ATT in form of isoniazid (INH), rifampicin, ethambutol and pyrazinamide along with tab pyridoxine, after CSF tap. She was also put on Inj dexamethasone $6 \mathrm{mg}$ four times a day. She responded to treatment and became asymptomatic and afebrile within a week of starting of treatment. After two weeks she was discharged with ATT and an advice for regular follow up. Steroid was stopped after three weeks. On routine OPD follow up maternal and foetal condition were normal. She underwent spontaneous labour at 37 weeks 04 days POG and delivered a normal male baby of $2.8 \mathrm{~kg}$ with normal APGAR at one and 5 minutes. Baby was screened for congenital tuberculosis and was then started on isoniazid chemoprophylaxis with an advice to take BCG vaccination after 6 months.

\section{DISCUSSION}

Tuberculosis is an important cause of maternal and perinatal morbidity. Out of 11 million cases worldwide, 2.6 million cases are there in India. ${ }^{6}$ The vast majority have latent rather than active TB. Of those infected 5$10 \%$ progress to active tuberculosis in their lifetime. Prevalence is more in HIV positive cases who carry an even higher risk of progression to active disease. The disease is responsible for killing more women of reproductive age than all the combined causes of maternal mortality. Exact data in respect of the proportion of pregnant women harbouring $\mathrm{TB}$ is unavailable in our country. However, it is presumed that incidence in pregnant mother is same as in general population. Pelvic TB leading to infertility in one third cases is more common in our country than CNS tuberculosis. ${ }^{1}$

Diagnosis of tuberculosis in pregnancy may be challenging, as the symptoms like lassitude, loss of appetite, nausea, vomiting and mild grade of fever, may initially be ascribed to the pregnancy, and the normal weight gain in pregnancy may temporarily mask the associated weight loss. ${ }^{7}$ Obstetric complications of TB include spontaneous abortion, preterm labour, low birth weight, and increased neonatal mortality. ${ }^{8}$ Congenital TB though quite rare, is associated with high perinatal mortality. Pregnancy per say doesn't affect the course of disease but associated poor nutritional state, immune compromised status or co-existing diseases may flare up tuberculosis. INH, Rifampicin, and Ethambutol are the first line drugs while Pyrazinamide use in pregnancy is gaining popularity and now recommended by World Health Organisation (WHO) for use in Tuberculosis. ${ }^{9}$ WHO recommends 6 months INH chemoprophylaxis for all babies born out of mothers suffering from Tuberculosis followed by BCG vaccination. ${ }^{9}$

Central nervous system involvement, one of the most devastating clinical manifestations of tuberculosis is noted in 5 to $10 \%$ of extra pulmonary TB cases, and accounts for approximately $1 \%$ of all TB cases. ${ }^{10}$ Mycobacterium tuberculosis is the major human pathogen. However in immunocompromised patients, atypical mycobacteria are an important cause of infection. 
History of tuberculosis is elicited in only approximately $10 \%$ of patients, as in our patient. ${ }^{10}$ Hematogenous spread seeds meninges or brain. Small tuberculous lesions (Rich foci) develop in meninges, subpial or subependymal surface of the brain or the spinal cord, and may remain dormant for years. Proximity to Sub Arachnoid (SA) space determines type of CNS involvement. Rupture into SA space leads to meningitis. Deep infiltration in parenchyma causes tuberculomas or abscesses. Paresis of cranial nerves is common as tubercular involvement is more at base of brain. Disease evolves over 1-2 weeks. Thick gelatinous exudate infiltrates the cortical and meningeal blood vessels, producing inflammation, obstruction, or infarction. Ultimately this may lead to coma, with hydrocephalus and intracranial hypertension. Fortunately, we had diagnosed our patient well in time, before complications could arise.

Clinical features of CNS TB include history of vague ill health for 2-8 weeks prior to development of meningeal irritation. The initial non-specific symptoms like headache, vomiting, low grade fever, malaise, anorexia and irritability may mimic normal pregnancy symptoms. Almost all these features were there in our patient. Later, with involvement of CNS the patient may present with severe headache, confusion, lethargy, altered sensorium and neck rigidity. On examination, depression of consciousness level, features of meningism, cranial nerve palsies-mainly occulomotor, papilloedema, and focal hemisphere signs may be present. Drowsiness, papilloedema and features of meningism were present in our patient.

Early diagnosis requires high index of suspicion. Nonspecific early symptoms of the disease may be mistaken for that of pregnancy, leading to missed or late diagnosis. We could suspect the problem in our patient because of previous history of incomplete treatment of TBM. Other causes (infective and non-infective) of TBM, cerebral malaria, late stages of dengue may be important differential diagnosis. All were negative in our case. Sporadic case reports of TBM, misdiagnosed as hyperemesis gravidarum, exist in the literature. ${ }^{11}$

Lumbar puncture is an essential step in the diagnosis. High leucocyte count (upto $1000 / \mu \mathrm{L}$ ) with lymphocytic predominance, elevated Protein (100-800 mg/dL), mildly decreased glucose concentration $(20-40 \mathrm{mg} / \mathrm{dL})$ are nonspecific findings. Smear is positive in only $10 \%$ cases. Culture is gold standard but is positive in $25-70 \%$. A raised ADA value (sensitivities 44 to $100 \%$ and specificities 71 to $100 \%$ ) points towards tubercular etiology. ${ }^{10}$ TB PCR may help in diagnosis $(56 \%$ sensitive and $98 \%$ specific) wherever facility exists. CT or MRI confirms the presence and extent of basal arachnoiditis, cerebral oedema, infarction, tuberculoma, ventriculitis and hydrocephalus. CT or MRI showing basal meningeal enhancement with any degree of hydrocephalus is strongly suggestive of TBM. In our case CSF suggested non-specific feature of TBM with negative slide and culture positivity for TB bacilli. Serum ADA however was positive. MRI brain suggested inflammatory granulomas.

Treatment should be initiated even on high index of suspicion. ${ }^{4}$ We started the treatment immediately after CSF tap, without waiting for the report of culture and MRI. With the exception of streptomycin, the first line anti-TB drugs are safe for use in pregnancy. The principles of treatment of tuberculosis in HIVseronegative pregnant patients (for presumed drugsensitive disease) are the same as for nonpregnant patients. Initial therapy includes INH, Rifampicin, Pyrazinamide and Ethambutol. ${ }^{9}$ Pregnant ladies have a high risk of developing INH induced peripheral neuropathy and should receive supplemental Pyridoxine at a dose of 10-25 mg/day for prophylaxis. ${ }^{9}$ With good response ethambutol and pyrazinamide may be discontinued after 2 months. But INH and Rifampicin should be continued for 6-12 months. However, there exists a controversy in total duration of treatment. Dexamethasone should be given in HIV negative patients @ 12-16 mg/day for 3 weeks, tapered over next 3 weeks. $^{12}$ Non-compliance is an important factor for treatment failure ${ }^{13}$ and our patient is a good example of it. Obstructive hydrocephalus and neurological deterioration may require ventricular drain or ventriculoperitoneal or ventriculoatrial shunt. Severe stage of disease with associated medical disorders especially HIV and delay in initiation of treatment are poor prognostic markers.

\section{CONCLUSION}

CNS tuberculosis is a serious but treatable disorder with protean manifestations. Early diagnosis requires a high index of suspicion, especially in pregnant patients. CSF cytology and biochemical analysis along with ADA gives valuable clue to the diagnosis. CT or MRI is useful in finding out tubercular CNS involvement. Clinical outcome depends greatly on the stage of disease at which therapy is initiated. Timely and appropriate initiation of treatment may have a favorable pregnancy outcome, as we could achieve in our case.

\section{Funding: No funding sources \\ Conflict of interest: None declared \\ Ethical approval: Not required}

\section{REFERENCES}

1. Parikh FR, Naik N, Nadkarni SG, Soonawala SB, Kamat SA, Parikh RM. Genital tuberculosis is a major pelvic factor causing infertility in Indian women. Fertil Steril. 1997;67:497.

2. D'Cruz IA, Dandeker AC. Tuberculous meningitis in pregnant and puerperal women. Obstet Gynecol. 1968;31:775-9.

3. Fallon RJ, Kennedy DH. Treatment and prognosis in tuberculous meningitis. J Infect (Suppl). $1981 ; 3(1): 39-41$. 
4. John CP. Ktngdom, Dermot H. Kennedy. Tuberculous meningitis in pregnancy. Br J Obstet Gynaecol. 1989 Feb;96:233-5.

5. Prevost MR, Fung Kee, Fung KM. Tuberculous meningitis in pregnancy - implications for mother and fetus: case report and literature review. J Matern Fetal Med. 1999;8:289.

6. World Health Organization. Global tuberculosis report, 2014. Available at: http://apps.who.int/iris/bitstream/10665/137094/1/97 89241564809 eng.pdf?ua=1.

7. M. Loto, Ibraheem Awowole. Tuberculosis in pregnancy. J Pregnancy. 2012;2012:379271.

8. Jana N, Vasishta K, Jindal SK, Khunnu B, Ghosh K. Perinatal outcome in pregnancies complicated by pulmonary tuberculosis. Int $\mathrm{J}$ Gynecol Obstet. 1994;44:119-24.

9. WHO. Guidelines for treatment for tuberculosis, 2010 . Available http://www.who.int/tb/publications/2010/978924154 7833/en/.

10. Cherian A, Thomas SV. Central nervous system tuberculosis. Afr Health Sci. 2011 Mar;11(1):116-27.

11. Kutlu T, Tugrul S, Aydin A, Oral O. Tuberculous meningitis in pregnancy presenting as hyperemesis gravidarum. J Matern Foetal Neonat Med. 2007 Apr;20(4):357-9.

12. Prasad K, Singh MB. Corticosteroids for managing tuberculous meningitis. Cochrane Database Syst Rev. 2008;(1):CD002244.

13. Sackoff JE, Pfieffer MR, Driver CR, Streett LS, Munsiff SS, DeHovitz JA. Tuberculosis prevention for non-U.S.-born pregnant women. Am J Obstet Gynecol. 2006;194:451-6.

DOI: $10.18203 / 2320-1770 . i j r \operatorname{cog} 20150127$

Cite this article as: Singh $\mathrm{S}$, Jose $\mathrm{T}$, Saxena $\mathrm{P}$. A rare case of CNS tuberculosis with pregnancy. Int J Reprod Contracept Obstet Gynecol 2015;4:911-4. 